\title{
La Superioridad Aérea en las políticas de seguridad y defensa de Suramérica*
}

| Fecha de recibido: 16 de octubre del 2019 | Fecha de aprobación: 29 de abril del 2020 |

\author{
David González-Cuenca \\ Magíster en Relaciones \\ y Negocios Internacionales \\ Docente e Investigador, Universidad \\ Militar Nueva Granada \\ Grupo de Investigación: PIREO \\ Rol de investigador: intelectual, \\ experimental, comunicativo \\ https://orcid.org/0000-0002-6563-9193 \\ $\triangle$ david.gonzalez@unimilitar.edu.co
}

\author{
Douglas Eduardo \\ Molina-Orjuela \\ Magíster en Desarrollo Rural \\ Docente, Pontificia Universidad Javeriana \\ Grupo de Investigación: GRIALI \\ Rol de investigador: intelectual, \\ experimental, comunicativo \\ https://orcid.org/0000-0003-0788-9538 \\ $\bowtie$ douglasemolina@gmail.com
}

* Este documento es producto del proyecto de investigación denominado "La importancia de la superioridad aérea para el Estado colombiano en el siglo xx", a cargo del Departamento de Fuerza Aérea y financiado por la Escuela Superior de Guerra. El proyecto está adscrito al grupo de investigación Masa Crítica.

Cómo citar este artículo: González-Cuenca, D., \& Molina-Orjuela, D. E. (2020). La Superioridad Aérea en las políticas de seguridad y defensa de Surámerica. Revista Ciencia y Poder Aéreo, 15(1), 71-86. https://doi.org/10.18667/cienciaypoderaereo.653 


\section{La Superioridad Aérea en las políticas de seguridad y defensa de Suramérica}

\section{Supremacy in South American Air Security and Defense Policies}

Resumen: El desarrollo de las políticas de seguridad y defensa en Suramérica durante el siglo xxI ha sido un tema crítico, particularmente por las dificultades socio-económicas y las transformaciones políticas que la región ha tenido que afrontar. Este artículo realiza un análisis comparativo de las diferentes políticas de seguridad y defensa de la región. Mediante ese análisis, se propone identificar las cercanías conceptuales o estratégicas en la consecución de la superioridad aérea (objetivo fundamental en los conflictos armados). Por medio de una metodología cualitativa con enfoque descriptivo y comparativo, se analizan las políticas públicas de Suramérica, haciendo énfasis en las capacidades estratégicas en el ámbito aéreo, lo cual permite la identificación de características propias de la superioridad aérea en la formulación de dichas políticas.

Palabras clave: Políticas públicas; seguridad y defensa nacional; superioridad aérea; Suramérica.

Abstract: The evolution of security and defense policies in South America during the 21st century has been a critical issue due to the socioeconomic difficulties and the political transformations experienced in this region. Therefore, this article presents a comparative analysis of the different security and defense policies established in South American countries with the aim of identifying conceptual or strategic similarities for achieving air supremacy, the main objective in armed conflicts. By means of a qualitative methodology with a descriptive and comparative approach, public policies of South American countries on this issue are analyzed, emphasizing strategic capabilities within the air sector. This analysis allows the identification of air supremacy features for the formulation of air security and defense policies.

Keywords: Public Policies; National Security and Defense; Air Supremacy; South America.

Resumo: $O$ desenvolvimento de políticas de segurança e defesa na América do Sul durante o século XxI tem sido uma questão crítica, principalmente por conta das dificuldades socioeconômicas e às transformações políticas que a região teve que enfrentar. Este artigo realiza uma análise comparativa das diferentes políticas de segurança e defesa na região. Por meio dessa análise, propõe-se identificar a proximidade conceitual ou estratégica na conquista da superioridade aérea (objetivo fundamental nos conflitos armados). Através da metodologia qualitativa, com uma abordagem descritiva e comparativa, são analisadas as políticas públicas na América do Sul, enfatizando nas capacidades estratégicas no setor aéreo. Esta análise permite a identificação de características de superioridade aérea na formulação destas políticas.

Palavras-chave: Políticas públicas; Segurança e defesa nacional; Superioridade aérea; América do Sul. 


\section{Introducción}

Desde la creación del avión, a principios del siglo xx por los reconocidos hermanos Wright, hasta la Teoría del Poder Aéreo, concebida por Alexander Seversky, la utilización de las aeronaves como parte de la fuerza militar de una nación ha sido crucial para lograr la superioridad frente al enemigo. Sin embargo, la verdadera demostración de una actividad estratégica fundamentada en un conjunto de aviones dispuestos para el combate bélico, se presentó solo hasta la Primera Guerra Mundial. Fue entonces, en la Gran Guerra, cuando se desarrollaron misiones de exploración aéreas que terminaron por definir su rumbo (Jordán Enamorado, 2016; Renouvin, 1990).

Las actividades desarrolladas por las fuerzas aéreas durante la Gran Guerra se enfocaron en aspectos estratégicos, tales como fotografiar la ubicación de las baterías de artillería y vigilar al enemigo para prevenir emboscadas $u$ ofensivas clandestinas. Sus acciones más importantes estuvieron relacionadas, no obstante, con el ataque a la infraestructura enemiga más importante: los depósitos de materiales y los equipos de aprovisionamiento de las líneas ofensivas y defensivas (Renouvin, 1990).

En ocasiones, la fuerza aérea era convocada a realizar operaciones contra las tropas de a pie. Estas maniobras generaban una tal efectividad que los comandantes consideraban insignificante el costo de operación de un ataque aéreo en relación con la posibilidad de obtener victorias tempranas y mermar la moral del enemigo con pocos recursos. Así mismo, la selección del personal de aviación se convirtió en un aspecto crucial dentro de las operaciones bélicas, puesto que el mal manejo de los aviones generaba pérdidas importantes, tanto monetarias como humanas, para el desarrollo de la guerra (Giaccaglia, 2017; Saíz \& Saíz, 2012).

Durante la Primera Guerra Mundial, la mayor efectividad del ejército alemán, que superó a la del ruso y el británico, se fundamentó en su capacidad aérea. Por su parte, el ejército ruso tenía una ausencia de aviación y el alemán, baja capacidad de reacción. A pesar de haber sido derrotados en la guerra, los avances alemanes en términos aéreos produjeron un progreso en el desarrollo estratégico del poder aéreo. En este sentido, el concepto de superioridad aérea se refiere a uno de los factores determinantes en el desarrollo de las guerras y, por tanto, un objetivo a conseguir para garantizar la victoria definitiva.

Este artículo pretende establecer la importancia que ha tenido el concepto de superioridad aérea en la formulación de las políticas de seguridad y defensa nacional de los países suramericanos. Para alcanzar este propósito, se presentará una conceptualización de lo que es la superioridad aérea, la importancia estratégica que esta reviste y el reconocimiento político que se le da por medio de su inclusión en las políticas nacionales de seguridad y defensa.

Se aplica en este artículo una metodología de tipo cualitativo con énfasis en el análisis comparativo de las políticas de seguridad y defensa de los países de Suramérica. Este estudio se centra en las políticas en las cuales se identifica el concepto de superioridad aérea o las funciones propias de la Fuerza Aérea relacionadas con la consecución de dicha superioridad.

\section{De la Superioridad Aérea a la Teoría del Poder Aéreo}

Los avances tecnológicos en el área de la aviación, en primera instancia militar, pero también comercial, han sido determinantes para el desarrollo de una visión estratégica en los conflictos armados y las guerras. Esta visión estratégica se sustenta en el rol de la fuerza aérea.

Como se mencionó anteriormente, el desarrollo de la aviación militar, consecuencia de la Primera Guerra Mundial, generó una serie de necesidades relacionadas con el desarrollo de nuevas características de motores, la determinación de la posibilidad de disparar un arma atrás de las hélices, la disposición de un fuselaje resistente a las condiciones de combate y la obtención de un mejor rendimiento del combustible (Corum, 2019; Grupo Simbólico de Transporte Aéreo, 2018). 
La utilización de una fuerza aérea por parte de Alemania durante la Primera Guerra Mundial, no solo trajo consigo un avance tecnológico en las herramientas a utilizar, sino que también mostró al mundo una nueva manera de realizar la guerra. Esto obligó a los estrategas a replantear sus posiciones y opciones en el frente de batalla y en la retaguardia. Debido a esta labor de los estrategas, se dio una superioridad de las fuerzas aliadas.

El concepto de superioridad aérea se refiere a la capacidad que tiene un actor de detectar mayores y mejores posibilidades en el campo aéreo, es decir, la capacidad de obtener y mantener una ventaja aérea frente al enemigo. El surgimiento del concepto de superioridad aérea representa para el campo de batalla la posibilidad de desarrollar estrategias de combate más efectivas, de menor despliegue de recursos humanos y de impactos más rápidos. Con esto, los resultados se pueden dar de un modo más eficiente y con menor desgaste.

Al finalizar la Gran Guerra, los ingenieros se enfocaron en desarrollar mejores capacidades de vuelo, lo cual representó el surgimiento de la aviación como una de las grandes industrias de la humanidad. Dentro de las capacidades de vuelo encontramos:

El alcance: en 1918 hasta los aeroplanos más livianos podían volar cientos de kilómetros; la velocidad: más de 150 km. por hora; altitud: la capacidad de volar sobre montañas, ríos y bosques, obstáculos que impedían el avance de las fuerzas de superficie; capacidad de fuego: la concentración de fuego podía dirigirse a puntos específicos en el frente de batalla o detrás de la línea enemiga; y flexibilidad: una combinación de otros atributos que permitían el uso de aeroplanos de forma rápida, en diferentes modalidades y lugares (Grupo Simbólico de Transporte Aéreo, 2018).

Así como evolucionaron las ventajas, también lo hicieron las dificultades para el desarrollo de estrategias de combate aéreo, lo cual resaltó las características negativas de este tipo de combate: la capacidad limitada para sostener un ataque por un tiempo mayor al dispuesto para las aeronaves; efectuar los ataques bajo condiciones climáticas adversas o en la noche; la dependencia de criterios políticos para la realización de los ataques, y la imposibilidad de capturar territorios o ejercer presión sobre los mismos. Todos estos son aspectos que, para la época, dificultaron el desarrollo de planteamientos de combate basados en la superioridad aérea. Sin embargo, estas características han cambiado y las dificultades han disminuido con el desarrollo de nuevas tecnologías.

En el ámbito estratégico, las capacidades aéreas en combate se usaron para desarrollar enfrentamientos entre aviones caza. Durante el periodo intermedio entre las guerras mundiales, estrategas como Douhet y Mitchell plantearon que las acciones dirigidas a estructuras físicas enemigas, según el primero, y a estructuras físicas, sumando el combate aéreo a estas acciones, según el segundo, son las mejores para la aplicación de la superioridad aérea (Grupo Simbólico de Transporte Aéreo, 2018).

A partir de la evolución del combate aéreo y las capacidades de lucha desde el aire, la Luftwaffe alemana estructuró una estrategia, durante la Segunda Guerra Mundial, basada en la conquista de la superioridad aérea. Esta estrategia no tuvo el resultado esperado, pues Alemania perdió la guerra. La estrategia se enfocó en desarrollar acciones ofensivas de corta duración, pero que causaran grandes afectaciones a la infraestructura enemiga, y en la disminución de poder bélico terrestre y aéreo. Así, se determinó como su objetivo principal la consecución de la superioridad aérea, debido a que esta daba posiciones de observación $y$ ataque determinantes para el avance de los frentes en combate (Andrews, 1995).

La Fuerza Aérea alemana tuvo victorias cruciales durante la Segunda Guerra Mundial, especialmente al enfrentar a las fuerzas británicas (antes de que la incursión de Estados Unidos de América diera una vuelta a la guerra en favor de las fuerzas aliadas). Según afirma el mayor Andrews, el manual de doctrina de operaciones del ejército alemán, decisivo para estas victorias, decía en 1934, "que para llevar a efecto operaciones terrestres exitosamente, uno debería 
buscar establecer superioridad aérea sobre el enemigo en el momento decisivo" (Andrews, 1995). Este fue el soporte para que, en el desarrollo de otras guerras, se pretendiera emular la acción de la Luftwaffe (alcanzar la superioridad aérea) como estrategia determinante para la finalización del conflicto. No obstante, también es importante resaltar que la fuerza aérea estadounidense alcanzó la superioridad aérea a partir de la Batalla del Paso Kasserine durante la Segunda Guerra Mundial, en las operaciones en Túnez (Serrano, 2017; Widnall, 1995).

Con la finalización de la Segunda Guerra Mundial, el mundo presenció un nuevo tipo de lucha que excedió las capacidades militares tradicionales. La bomba atómica, arrojada por Estados Unidos sobre Japón desde un Boeing B-29 llamado Enola Gay (en tributo de la madre del piloto), pronosticó un rumbo diferente al que hasta ese día se había dado en las confrontaciones bélicas. La rivalidad latente entre Occidente y Oriente permitió a los analistas y pensadores de la guerra hacer un alto en el camino y visualizar el panorama. En 1950, el militar ruso Alexander Seversky inició el estudio del poder aéreo. En su indagación se determinaba que "el poder marítimo y el poder terrestre estaban en equilibrio por la guerra fría" (Giudice, 2005, p. 21). Con esta teoría, Seversky presenta una forma de eliminar las barreras en tierra, sobrepasar los denominados “Estados tapón” y controlar decididamente puertos, rutas y mares (Zuinaga, 2015).

Con la construcción de la Teoría del Poder Aéreo, Seversky dividió el mundo en tres grandes zonas aéreas:

1. La zona aérea de Estados Unidos y su espacio de reserva: América del Norte, Central y del Sur. Las tres Américas son consideradas como las reservas geográficas de la industria de Estados Unidos.

2. La zona aérea de la Unión Soviética que se proyecta hasta el África como reserva. Considera a China como zona de influencia que la distraerá hacia el Asia.

3. Zona de decisión aérea: los espacios industriales de ambas potencias; Estados Unidos y la Unión Soviética (Giudice, 2005).
A partir de la construcción que hace el teórico ruso sobre las zonas aéreas de relevancia, y de cara a nuestro estudio, debemos destacar la primera zona, la cual vincula a América del Norte con América Central y del Sur. Esta vinculación es reafirmada por el desarrollo geoestratégico que presentó Estados Unidos a partir del New Deal, por la política de buena vecindad fomentada hacia América Latina desde mediados del siglo xx, e incluso por la exposición de una política de seguridad y defensa hemisférica de la mano de la Doctrina Truman y la lucha contra el narcotráfico, que se mantiene hasta hoy.

La aviación y las capacidades bélicas aéreas en América Latina han tardado en desarrollarse. Sin embargo, el avance decidido de la aviación argentina, chilena, brasileña y colombiana, esta última por cuenta de diferentes conflictos, ha sido relevante para considerar de vital importancia un análisis de las condiciones de superioridad aérea en la región. La industria de aviación militar en Brasil, una de las más desarrolladas del mundo, diferentes hitos establecidos por la participación de la Fuerza Aérea de Colombia en el conflicto armado interno, y las relaciones de Venezuela con Rusia en términos del abastecimiento de flotas aéreas de gran capacidad, son factores que se deben analizar desde el punto de vista de la toma de decisiones. Es decir, deben analizarse las estrategias, actividades o doctrinas al interior de cada país como parte de la formulación de las políticas de seguridad y defensa nacionales enfocadas en lograr la disuasión ${ }^{1}$ y contención mediante la superioridad aérea.

1 Se considera que la disuasión es una de las más importantes teorías de la seguridad y defensa nacional, categoría que se encuentra en los estudios de relaciones internacionales y que ha sido promovida por Estados Unidos a partir de la creación y utilización de armas termonucleares de destrucción masiva. El concepto de disuasión está relacionado con la prevención de conflictos armados y la demostración de poder frente al contrincante. En este sentido, la disuasión se define como "[...] la amenaza de recurrir a la fuerza, en proporción capaz de causar daños difícilmente asumibles, con el objeto de evitar un ataque" (Reyes Segovia \& Urbina Rodríguez, 2014, p. 36, citando a Sodupe, 1991). 


\section{Las políticas de seguridad y defensa nacionales en Suramérica}

El desarrollo de las políticas públicas en Suramérica, por tradición y con contadas excepciones, se ha presentado en un marco de dependencia con respecto a Estados Unidos, como máximo influenciador. Su influencia viene de la consecución de su estatus de potencia mundial tras la Segunda Guerra Mundial. Hay que tener todo lo anterior en cuenta, especialmente si se pretende analizar las políticas de seguridad y defensa, principales intereses del país del Norte de la región.

A partir de 1945, la política exterior estadounidense fijó su mirada en Suramérica. En particular, esta política estaba guiada por las pretensiones de ejercer control sobre la región para evitar la expansión del comunismo en el hemisferio occidental. Con este interés, se propagó un discurso pan-americanista que tuvo su demostración en la renombrada Ix Conferencia Panamericana, realizada en Bogotá, Colombia. En la conferencia se firmó la carta de la Organización de Estados Americanos, que dio origen a dicha organización internacional en 1947.

En el transcurso de la Guerra Fría, y como resultado del fuerte poder económico adquirido por Estados Unidos al terminar la Segunda Guerra Mundial, su política exterior se basó principalmente en controlar de manera política y disciplinar a los gobiernos de América Latina (Aguirre, 2006). Sus intereses en cada uno de los países variaron de tiempo en tiempo. Como nunca antes, los países del hemisferio se comprometieron de diversas maneras a ofrecer su apoyo incondicional a la potencia del Norte en caso de una guerra con la Unión de Repúblicas Socialistas Soviéticas (URSS). La confrontación diplomática, y en ocasiones la confrontación militar con Cuba y algunos países centroamericanos, se mantuvieron como constante. En todo este proceso de confrontación, Estados Unidos tuvo como máximo respaldo a Colombia, socio-estratégico y reproductor de los intereses del gigante del Norte en la región.

Debido a la lucha contra el comunismo, la influencia estadounidense en la región estuvo determinada por la participación de fuerzas de inteligencia. Tales son los casos del ascenso de Augusto Pinochet al poder en Chile o la invasión a Bahía de Cochinos, entre otros. Estas acciones propiciaron el surgimiento en algunos gobiernos con marcadas políticas anti-norteamericanas y anti-imperialistas que pretendían, y pretenden aun en la actualidad, determinar políticas de seguridad y defensa no adscritas al criterio de Washington. En ocasiones, la reticencia de otros gobiernos, que no quieren alejarse de dicho paradigma, ha provocado distorsiones en las relaciones y la seguridad de la región (Peixoto de Oliveira, 2014).

Pero el comportamiento de las agendas de seguridad y defensa en la región suramericana ha venido evolucionando. Con la llegada del siglo xxı, muchos gobiernos han optado por la modernización de sus tropas y equipos, y han puesto un gran énfasis en las fuerzas de aire, interpretando que ellas proveen una ventaja en teatros de confrontación bélica. Este énfasis también se debe a comportamientos macroeconómicos importantes, como el incremento del nivel de renta proveniente de la explotación de hidrocarburos y de la minería.

Las economías de Suramérica son más ricas actualmente, a pesar de los altos índices de desigualdad que presenta la región (Fitzgerald, 2009). Adicionalmente, "los ingresos per cápita son considerablemente más altos de lo que eran a comienzos o mediados del siglo pasado" (Sanguinetti \& Villar, 2012). Lo anterior ha favorecido el incremento del gasto en defensa por parte de los gobiernos. Los constantes reproches de los ciudadanos por este gasto, sin embargo, han privado a los países de la posibilidad de adquirir más y mejores equipos de alta tecnología para el desarrollo de combates aéreos, o para repeler los mismos.

A partir de la anterior conceptualización, y resaltada la importancia que tiene la superioridad aérea como parte fundamental de los objetivos de una política de seguridad y defensa nacional en términos de contención y disuasión, se realizará un análisis de las políticas en Suramérica y de las capacidades que ellas dan a sus fuerzas aéreas para asegurar la superioridad aérea: 


\section{Argentina}

Con el final de la dictadura y el comienzo de un nuevo milenio, se dio en Argentina una discusión en torno a cuál sería el papel que jugarían las fuerzas armadas en la protección de las fronteras nacionales y la población. Esta discusión llevó al establecimiento de varias leyes y decretos que determinaron la política militar del país, la cual incluye a las fuerzas aéreas. Durante dicho periodo, la nación del Sur tenía una fuerza aérea en deterioro, pequeña en comparación con la calidad y cantidad de material aéreo de combate disponible. Sin embargo, la Fuerza Aérea argentina ha podido ser gestionada, gracias a la alianza del país con la OTAN, con equipos que de alguna manera no han sido tan efectivos por causa de la crisis económica (Pizarro, 2002; Piglia, 2018).

No obstante, Simonetta (2016) afirma que, ante el fin de la actividad de los aviones supersónicos, "el servicio de defensa del espacio aéreo argentino quedó sin prestación". Esto a pesar de que en el 2001 se realizaron los operativos Escudo Norte y Fortín I y II, cuyo objetivo era incrementar la vigilancia y el control de los espacios aéreos, fluviales y terrestres en las fronteras del norte argentino. Para ello, se usaron radares y recursos militares en articulación con las fuerzas de seguridad (Facultad Latinoamericana de Ciencias Sociales, 2016).

Con relación a la política pública de seguridad y defensa nacional, Argentina cuenta desde 1998 con el Libro Blanco de la República Argentina. En este libro no se encuentra ninguna referencia directa o indirecta a la construcción de superioridad aérea. Sin embargo, las funciones que se le otorgan a la Fuerza Aérea se presentan dentro de categorías genéricas como la vigilancia y control del espacio aéreo o la defensa y el desarrollo de misiones estratégicas, tácticas y de transporte (Ministerio de Defensa Nacional de Argentina, 1998).

Por último, como se mencionó anteriormente, la participación militar interna en Argentina ha sido motivada por la potencia del Norte. No obstante, esta potencia ha replanteado en la actualidad sus prioridades, enfocándose en amenazas internas tales como el terrorismo y el narcotráfico; esta última cuestión se considera la más importante.

\section{Bolivia}

El país andino es uno de los tantos que ha escatimado esfuerzos y recursos para conseguir una flota de combate aéreo importante. Gran parte de su flota proviene del apoyo de Estados Unidos, que les ha brindado helicópteros y otras naves con el objetivo de combatir el narcotráfico (Pizarro, 2002).

Para el 2004, el Gobierno boliviano expidió el Libro Blanco de Defensa, el cual se sustenta en criterios de disuasión, cooperación, prevención y persuasión. Este libro, más allá de presentar el marco histórico de la creación de la Fuerza Aérea con la pretensión de consolidar un poder aéreo nacional, no hace mayor referencia al objetivo de la superioridad aérea. Las actividades básicas de la Fuerza Aérea boliviana son el ejercicio de caza y táctico, así como labores de transporte y de policía (Ministerio de Defensa de la República de Bolivia, 2004).

Cabe resaltar que el Ministerio de Defensa de Bolivia (2004) muestra en el documento un gran reconocimiento a la labor que, en términos de desarrollo nacional, ha realizado la Fuerza Aérea boliviana. Esta es presentada como un gran apoyo para las actividades de integración de las regiones más apartadas, dadas las condiciones adversas de la geografía.

\section{Brasil}

La política de seguridad y defensa brasileña, plasmada en el Libro Blanco del 2012, es una de las más completas de la región, pues refleja la concepción estratégica y militar utilizada actualmente por la potencia regional. Esta política se caracteriza por incluir elementos innovadores, expresados en un mayor impulso político y transparencia en los objetivos dispuestos. Esto se suma a una tradición marcada por la persistente influencia del pensamiento militar en áreas particulares (Vitelli, 2015). 
Dentro de la política de seguridad y defensa, la fuerza aérea ha sido uno de los ámbitos más relevantes. Brasil cuenta con una importante industria aeronáutica militar, una de las más poderosas en el hemisferio. Además, hay una articulación con los órganos de gobierno y los poderes políticos, la cual ha sido fundamental para generar una política coherente y pertinente para la realidad del país y el contexto internacional en el que se desenvuelve.

La cooperación para el desarrollo de tecnologías de combate aeronáutico, por ejemplo, con Sudáfrica y Paraguay, ha sido de gran importancia en los planteamientos políticos y militares del gobierno. Así mismo, ha sido central el desarrollo de acciones estratégicas en el sector espacial. Por tal razón, el país ha desarrollado una política nacional de desarrollo aeroespacial (Gobierno de la República Federativa de Brasil, 2012).

Al igual que otros países de la región, el concepto de superioridad aérea no aparece en la caracterización de los objetivos de importancia en la política brasileña. Sin embargo, la política brasileña es una de las pocas del hemisferio que contempla no solo la posesión de equipos, sino un planteamiento táctico-estratégico para la defensa de amenazas aéreas mediante la implementación de baterías antiaéreas al mando de una División Aérea y un Batallón Aero-táctico. Igualmente, más allá de plantear operaciones conjuntas, se desarrolla una política para llevar a cabo ejercicios de defensa naval para contrarrestar amenazas aéreas (Hadmann, 2019; Gobierno de la República Federativa de Brasil, 2012).

En función de la dimensión del país, la Fuerza Aérea de Brasil tiene un objetivo bastante complejo: asegurar y garantizar la protección del territorio. Esto ha requerido un desarrollo técnico, tecnológico, táctico y estratégico reflejado en el Libro Blanco de Defensa Nacional. Es loable que, en este marco, los escenarios de la política se planteen de manera coherente y específica frente a las amenazas que internacionalmente se presentan como retos. Por tal razón, la política brasileña otorga la importancia pertinente a la fuerza. Esto permite pensar que se identifica implícitamente a la superioridad aérea como una herramienta clave en de la formulación de la política pública.

\section{Chile}

Para el 2010, el Gobierno chileno publicó el Libro Blanco de la Defensa. A diferencia de otros países, en este libro se establecen concretamente las pretensiones de garantizar la seguridad del territorio a través del reconocimiento del espacio aéreo por controlar y de la función de la defensa aérea. En esta política se presenta por primera vez en la región una posición clara con respecto al control de la Antártida. Para ello, se establecen bases y estaciones operadas en conjunto por las Fuerzas Armadas, lo que fortalece la seguridad y el control sobre las rutas transpolares (República de Chile, 2010).

La necesidad de contar con una infraestructura terrestre, como apoyo a las operaciones aéreas, que sea acorde con las condiciones geográficas longitudinales del país se presenta como un factor estratégico relevante. En esto, la política chilena difiere de otras que conciben a la Fuerza Aérea como un apoyo de las fuerzas de tierra. Chile establece así un precedente para analizar el entendimiento y desarrollo de la superioridad aérea en Suramérica.

Según lo establece el Libro Blanco de la Defensa en el capítulo XIII, el Gobierno chileno considera que la Fuerza Aérea interpreta, dentro del teatro de operaciones, el papel de consolidación de una capacidad fundamentada en el poder aéreo. Este poder

pone el acento en el elemento de fuerza, pero se trata de un concepto que participa de la noción más general de "poderío aeroespacial" nacional y, por lo tanto, la función de control también asume un alcance mayor que el control operacional (República de Chile, 2010, p. 179).

El control aeroespacial es un concepto fundamentado en el concepto de poder aéreo que, como ya se mencionó, se basa en la consecución de la superioridad aérea. Esto nos permite determinar que hay una relación directa entre este concepto estratégico y las políticas de la nación austral. Por esta razón el documento mencionado tiene un acápite completo sobre el 
entendimiento del concepto de poder aéreo y su aplicación en el territorio nacional chileno.

El "poder aéreo" es el elemento de fuerza del poderío aeroespacial que se emplea en el espacio de batalla para hacer uso de este en beneficio propio y negárselo al adversario. Está representado por la suma de los sistemas de armas que emplea la Fuerza Aérea para combatir al enemigo e incluye todas las actividades de apoyo necesarias para su empleo y el personal instruido para operarlos (Siminic Ossio, 2018).

El poder aéreo tiene la capacidad de actuar en la profundidad del espacio de batalla. Es decir, por sus características, el poder aéreo puede realizar operaciones individuales, en el sentido de que sólo implican medios aéreos, pero en apoyo al objetivo estratégico conjunto final. Entre las características del poder aéreo se destacan la capacidad de graduar su aplicación, su rápido despliegue a teatros distantes, la posibilidad de potenciar las fortalezas de las fuerzas propias y explotar las debilidades del adversario.

El poder aéreo está constituido por elementos que permiten potenciar sus resultados. Algunos de ellos son los siguientes: un sistema logístico capaz de apoyar a la fuerza en forma sostenida, oportuna, en la cantidad y con la calidad requerida; una inteligencia precisa y oportuna para la definición de la estrategia aérea y la correcta selección de blancos; finalmente, un sistema de mando y control que permite el mando único y el control centralizado para la ejecución descentralizada de las operaciones aéreas.

Se destaca que las condiciones para realizar operaciones conjuntas, gracias al desarrollo de actividades aéreas, muestran el desarrollo chileno en comparación con la región. En las actividades aéreas, la aviación naval, de ejército y la Fuerza Aérea cooperan decididamente en el desarrollo de operaciones expedicionarias. Se destaca que la aviación naval ha desarrollado, al igual que en Brasil y Argentina, una escuadra de ataque con presencia internacional basada en el poder naval. Esta escuadra se puede extender hacia la región de Asia y el Pacífico (Pizarro, 2002).

\section{Colombia}

La participación del componente de defensa nacional en el presupuesto colombiano, ha sido determinada por las particularidades del país en seguridad interna (con un constante conflicto armado) y por las repercusiones que la situación de seguridad nacional ha tenido en la región y en el hemisferio (especialmente debido a la expansión del narcotráfico). Estas condiciones han sido determinantes desde finales del siglo xx, lo que ha propiciado un incremento constante en el presupuesto destinado a la defensa nacional desde ese entonces.

La participación o cooperación con países fronterizos para apoyar la lucha contra los dos principales problemas de seguridad ha sido poca. La región no se ha involucrado directamente en apoyos o acciones militares conjuntas para abordar las amenazas, que son compartidas en muchos escenarios. La excepción más notable es Estados Unidos, que, por cuenta de sus pretensiones en la región, ha tenido a Colombia como principal socio en la seguridad hemisférica de la región.

A partir de 1999, debido a las relaciones bilaterales entre Colombia y Estados Unidos, y las posibilidades que estas relaciones generaban para expandir su poder en términos de seguridad y defensa nacional, Colombia empezó a recibir un apoyo económico para la lucha contra el narcotráfico; este apoyo se denominó Plan Colombia. Con el Plan, se fortalecieron las capacidades militares, se establecieron protocolos de formación y se desarrollaron nuevos procesos enfocados en la finalización del conflicto. Desde ese momento, el conflicto quedó relacionado con el narcotráfico, por ser esta la principal fuente de financiación de los grupos alzados en armas.

El Plan Colombia pone entonces su atención en el narcotráfico. Se relaciona así esta problemática con el surgimiento y permanencia de grupos al margen de la ley. Según Ochoa (2013), esto termina por simplificar las causas del conflicto interno armado y desconoce las debilidades sociales del país, tales como la exclusión política y la inequidad; estas últimas hacen parte de las causas que dieron origen al conflicto. En 
consecuencia, siguiendo su motivación principal, el Plan desarrolla un programa para la erradicación de cultivos ilícitos, además de otras acciones que no guardan relación directa con la superioridad aérea.

Para el 2011, el Ministerio de Defensa Nacional de Colombia publicó la Política Integral de Seguridad y Defensa para la Prosperidad. Esta es una aproximación de política pública en el tema de seguridad y defensa nacional, pero no ahonda en la construcción estratégica o conceptual de proyecciones doctrinales para las actividades de las fuerzas armadas ante las amenazas latentes. Más allá de ser un documento preliminar, ligado a una política gubernamental, y que demarca el escenario para un futuro planteamiento de una verdadera política de seguridad y defensa nacional, esta política no presenta mayores aportes para enfrentar las condiciones de seguridad que vive la nación colombiana.

Aunque Colombia carece de una política de seguridad y defensa nacional, el documento comentado presenta dentro de sus objetivos y estrategias sectoriales un factor importante para el eventual desarrollo de una política que tenga la superioridad aérea como objetivo principal: "avanzar hacia un sistema de capacidades disuasivas creíble, integrado e interoperable" (Ministerio de Defensa Nacional, 2011). Esto abre el camino para desarrollar capacidades que garanticen la soberanía, y para desarrollar el combate con el objetivo de lograr la superioridad aérea.

Más allá de la política documental, después de la implementación del Plan Colombia, el país tiene una de las mejores flotas de helicópteros de la región para la lucha contra el narcotráfico, y también ha conseguido aviones de reconocimiento, ataque y control. Todos estos logros contribuyen al mejoramiento de las capacidades aéreas, las cuales son uno de los eslabones de la superioridad aérea. Sin embargo, el grueso de los equipos que componen la defensa aérea está a punto de finalizar su ciclo de uso. Por esta razón es necesario iniciar una renovación completa de la flota (Pizarro, 2002).

Una de las versatilidades de tener un poder aéreo fortalecido en un país como Colombia, es que posibilitará la capacidad de llegar a los rincones más alejados de la geografía Colombiana. Al hacer este tipo de ejercicios, mejoraría las relaciones cívico-militares, mediante el acceso a brigadas de salud y logística de transporte de alimentos y medicamentos. Este tipo de acciones, sin lugar a dudas mejoraría la confianza y el tejido social de la sociedad civil con el estamento militar.

\section{Ecuador}

En el 2005, Ecuador publica el Libro Blanco del Ecuador. Este se refiere puntualmente a la relación entre la política de defensa nacional y la política exterior, fomenta la cooperación en función de la paz y la seguridad del territorio. Como objetivo estratégico, se resalta la necesidad de ejercer control del espacio aéreo a partir del desarrollo de operaciones aéreas, tanto de combate como de transporte, rescate y defensa aérea (Tapia \& Gavino, 2019; República del Ecuador, 2005).

El Libro Blanco del Ecuador no contiene referencia alguna al concepto de superioridad aérea. No obstante, en el aspecto táctico se reconoce a la Fuerza Aérea como una de las más profesionales de la región, especialmente tras la victoria de esta fuerza sobre Perú en la denominada Guerra del Cóndor. A pesar de ello, esta guerra tuvo un aspecto negativo, pues conllevó a un embargo de armamento por parte de Estados Unidos, lo que limitó la evolución aérea del Ecuador que le pudiera garantizar la superioridad aérea (Pizarro, 2002).

\section{Paraguay}

La primera política de seguridad y defensa de toda la región es la Ley 1337 de 1998 de Defensa Nacional y Seguridad Interna del Paraguay. En esta ley se establecen los objetivos y recursos para garantizar la seguridad nacional. Se incluye la política militar y se tienen en cuenta "la totalidad de las potencialidades nacionales e institucionales, con relación a las necesidades de la defensa nacional" (Congreso de la Nación Paraguaya, 1998).

Lo más novedoso de esta ley es la creación del Consejo de Defensa Nacional. Este es el principal órgano asesor y consultivo para afrontar las situaciones de 
defensa nacional, sin desconocer la facultad principal del presidente como decisor de la política. Aunado a ello, se reconoce la movilización nacional como aquellas "previsiones y acciones emprendidas por el Estado durante la vigencia del Estado de Defensa Nacional con el objeto de optimar el poder nacional requerido para la defensa nacional" (Congreso de la Nación Paraguaya, 1998). Esta movilización es un bastión para el desarrollo de las potencialidades integradas de la nación en función de su defensa, y vincula tanto a la Fuerza Armada como a las instituciones públicas y a los ciudadanos en torno a un interés nacional de defensa, en caso de un conflicto armado internacional.

Esta ley hace evidente la importancia que el Estado da al control naval, en términos del desarrollo de capacidades de la Armada y los objetivos de navegabilidad de sus ríos. Esto deja entrever una primacía de los objetivos náuticos sobre los aéreos. Sin embargo, esta primacía no siempre se aparta del concepto de superioridad aérea, pues en el ejercicio de acciones conjuntas, y debido a una geografía conformada ríos y mares, la cooperación entre las fuerzas aérea y naval es crucial para su buen término, y eventualmente para la consolidación de la superioridad aérea.

Pizarro (2002) resalta la precariedad en la que se encuentra la Fuerza Aérea paraguaya. Destacar, sin embargo, que el interés por cambiar esta situación ha crecido recientemente y se ha venido trabajando para adquirir una superioridad aérea mediante la compra de diferentes aeronaves de gran capacidad bélica. La compra de estas aeronaves, en sí misma, no garantiza una mejora inmediata de la fuerza aérea, más aún si se carece de un ordenamiento de presupuesto coherente con las pretensiones legales. Sumado a esto, la clase dirigente del país no da un gran respaldo al desarrollo de capacidades aéreas.

\section{Perú}

Desde la expedición y posterior publicación del Libro Blanco de Defensa Nacional del Perú, en el 2004, ha habido múltiples avances en la construcción de políticas públicas en torno a la seguridad y la defensa. El Libro Blanco peruano es el marco normativo y estratégico que organiza al sector de defensa. Representa los intereses de la nación de proteger el territorio, garantizar la soberanía y la seguridad de la población. Dentro de los aspectos más relevantes de esta política marco, está el desarrollo de la Reserva Aérea Nacional y la delegación de actividades tendientes a establecer capacidades en el escenario aeroespacial a la Fuerza Aérea (Navarrete Anderson, 2019; Ministerio de Defensa del Perú, 2005).

Al igual que en otros países de la región, el narcotráfico ha determinado muchas de las acciones de desarrollo de capacidades militares. Perú ha puesto un especial énfasis en la consecución de capacidades aéreas en función de la interdicción aérea de las actividades del narcotráfico. De igual manera, Perú ha prestado una gran atención al análisis y la proyección de capacidades aéreas y antiaéreas. Esto nos presenta una aproximación a la concreción de la superioridad aérea desde la planeación estratégica, que apoya la reestructuración y modernización del Estado.

Cabe señalar que el Libro Blanco de Defensa Nacional determina puntualmente las capacidades de la Fuerza Aérea. Estas funciones incluyen el control aeroespacial, la superioridad de la información, las operaciones de precisión y la movilidad aérea eficaz; en conjunto, tienen el objetivo de dar al Estado una gran posibilidad de lograr la superioridad aérea por cuenta de la implementación táctica de los recursos estratégicos y de la adaptabilidad material de la fuerza bélica en un escenario de conflicto (Ministerio de Defensa del Perú, 2005).

La participación de la Fuerza Aérea Peruana en ejercicios de cooperación y en acciones conjuntas internacionales es destacable. Además, se resalta la participación en conflictos internacionales como el acaecido en Irán e Iraq a finales de la década de 1980, en Namibia en la misma época, en Sierra Leona en el 2000 y en Haití, con cuarenta y tres efectivos de la Fuerza Aérea. También se destaca la participación de este país en ejercicios de cooperación, como las operaciones UNITAS; ejercicios con Brasil, Chile y Ecuador en "detección, identificación e interceptación de aeronaves, ejercicios combinados para atender a la población en caso de desastres naturales y ejercicios de búsqueda y rescate" (Ministerio de Defensa del Perú, 2005). 
La evolución de la política pública peruana de seguridad y defensa nacional es de las más destacadas de la región. Si bien el Libro Blanco no fue publicado recientemente, el Estado ha seguido desarrollando ejercicios de actualización por medio de la expedición de varios decretos supremos y planes. En el 2011, por ejemplo, se expidió el Plan Estratégico de Desarrollo Nacional. Este plan vincula los conceptos de operatividad y eficacia del sistema planteado en el Libro Blanco para garantizar la defensa de los intereses nacionales (Presidente de la República del Perú, 2018).

En el 2007, se promulgó el Decreto Supremo 027. Dentro de sus lineamientos más relevantes, este decreto establece la participación activa de diferentes sectores en el cumplimiento de los objetivos de la política de seguridad y defensa. El Decreto Supremo 027 se apoya en el Decreto Legislativo 1129, que formula la creación y funcionamiento del Consejo de Seguridad y Defensa Nacional y lo presenta como el órgano decisivo principal en el sector. Todo lo anterior fue aprobado por el Decreto Supremo 012 del 2017.

Mediante este último decreto, el Estado peruano establece como principales objetivos la protección de la Amazonía, la presencia en la Antártida y la lucha contra la minería ilegal. Incluye además las luchas contra el terrorismo y el narcotráfico como ejes de acción estratégicos. Como aspecto diferenciador, esta política presenta una posición formal con respecto al concepto de identidad nacional. Presenta además un marco ampliado, multidimensional, del ejercicio de la seguridad y la defensa nacional, en el cual se articulan conceptos como el desarrollo económico, la lucha contra la pobreza, la desnutrición infantil y los conflictos sociales, entre otros. Esta articulación representa una verdadera innovación en comparación con otras políticas de la región (Presidente de la República del Perú, 2018).

La relación entre, por una parte, los sectores de seguridad y defensa y, por otra parte, la política y la rama judicial se destaca en la política del Perú. Esta política establece acciones determinantes para garantizar el logro de los objetivos de interés nacional, amenazados por la corrupción. En cuanto a la capacidad y superioridad aérea, no se hace referencia a esta en el documento.
En la misma línea, el presidente de la red táctica Consulting Services de Washington, José Miguel Pizarro Ovalle (2002), presenta a Perú como "probablemente la fuerza aérea de combate más poderosa de la región” en su informe "Poder Aéreo en América del Sur". Este juicio se basa en las condiciones de la flota de combate aéreo y los equipos de inteligencia aérea y baterías antiaéreas que posee Perú, provenientes de Rusia en su mayoría. Así pues, la Fuerza Aérea del Perú, junto con la de Brasil, se encuentra en la cima en cuanto a las posibilidades de obtención de superioridad aérea en la región.

\section{Uruguay}

La Política de Defensa Nacional del Uruguay, formulada en el 2014, se plantea a raíz de la política de gobierno denominada Un Uruguay integrado en la región y abierto al mundo, liderada por el presidente José Mujica. En este documento se pretenden integrar los conceptos de seguridad y defensa a los objetivos de desarrollo de la nación. Se plantean así objetivos a mediano y largo plazo con la vocación de construir una política estatal de seguridad y defensa nacional.

En ese sentido, el gobierno y las instituciones del Estado hacen un gran esfuerzo por vincular a la ciudadanía y a los diferentes actores sociales en el logro del objetivo común (la defensa nacional). La Política fue materializada por medio de la Ley Marco de Defensa Nacional n. ${ }^{\circ} 18650$. Allí se plasman los diferentes intereses nacionales que se quieren proteger con la eficiencia en la utilización de los recursos a disposición y con el desarrollo de potenciales multidimensionales (Gobierno de la República Oriental del Uruguay, 2014).

Como aspecto diferenciador del resto de las políticas aquí analizadas, la del Uruguay presenta un perfil absolutamente integrador con la región. Este perfil viene no solo del establecimiento de la política gubernamental de donde nace, sino de la filosofía propia de la política uruguaya. En ella, la seguridad regional se presenta como la base articuladora de la seguridad pública y la defensa militar. Se defiende que "la integridad territorial, la soberanía, y la paz del Uruguay, demandan un escenario de estabilidad y equilibrio en las relaciones interestatales" (Gobierno de la República 
Oriental del Uruguay, 2014). A partir de este postulado se fomenta la cooperación estratégica con las naciones de la región y se determina que este es el fundamento de la seguridad y la defensa nacional.

En esta política se presenta acertadamente la consideración de que las amenazas pueden venir del ámbito internacional. Por ello es necesario incentivar la cooperación interestatal para el desarrollo de procesos de integración con el fin de mantener la seguridad de la nación y de la región. Esto garantiza la salvaguarda de un espacio común (Gobierno de la República Oriental del Uruguay, 2014).

Por tanto, la República del Uruguay presenta una disposición de acción muy cercana a la Unión de Naciones Suramericanas (UNASUR). Sin embargo, desde su creación, las políticas nacionales no han reflejado la unidad que esta Unión supondría. En todo caso, se puede identificar un marco de diálogo al interior del Consejo de Defensa Suramericano (CDS). Esta institución permitirá afianzar los lazos de cooperación entre los ministerios de defensa de la región y construir agendas de seguridad y defensa comunes. En este sentido, la Política de Defensa Nacional del Uruguay califica al CDS como "de alto valor geopolítico por su impacto geoestratégico para la preservación de los recursos naturales estratégicos y la biodiversidad y la consolidación de Sudamérica como zona de paz y cooperación (Gobierno de la República Oriental del Uruguay, 2014).

Más allá de resaltar los valores tácticos o estratégicos de la operatividad de las fuerzas armadas, esta política presenta como valores superiores los escenarios de integración en el campo de la seguridad. Estos valores están representados en la creación del Centro de Estudios Estratégicos de la Defensa o en la creación de una industria militar regional. En este documento no se presentan criterios referentes a la superioridad aérea, sino que se caracterizan puntualmente los intereses nacionales, las amenazas y los escenarios que pueden amenazar dichos intereses.

\section{Venezuela}

La Asamblea Nacional de la República Bolivariana de Venezuela, expidió en el 2002 la Ley Orgánica de
Seguridad de la Nación. Esta ley se fundamenta en los principios de integralidad del desarrollo y la seguridad multidimensional. Se destaca la vinculación de la sociedad civil, considerando las diferentes condiciones sociales de sus pobladores, y la inclusión de los pueblos indígenas, incluso. Este es un aspecto que otras naciones no consideran (Asamblea Nacional de la República Bolivariana de Venezuela, 2002).

En sus planteamientos no hay una referencia clara a las condiciones, características o disposiciones que deben cumplir las Fuerzas Armadas. Tampoco se entrevé una pretensión de lograr la superioridad aérea como eje central de la estrategia de seguridad y defensa nacional. En este sentido, no existe mucha claridad sobre el poder aéreo de la Fuerza Aérea venezolana, especialmente con la situación socio-política que afronta este país desde hace ya varios años.

Sin embargo, la adquisición de diferentes aeronaves de alta capacidad bélica, por las fuertes relaciones entre Rusia y Venezuela en las últimas dos décadas, ha permitido a este país reforzar y fortalecer su capacidad aérea. Venezuela muestra un comportamiento tendiente a la consecución de la superioridad aérea, especialmente frente a Colombia y a Estados Unidos, países a los que la presidencia venezolana considera como la principal amenaza a su seguridad nacional.

\section{Conclusiones}

En términos generales, se puede observar que el desarrollo de políticas públicas de seguridad y defensa nacional en América del Sur ha sido poco. Pese a que las estructuras de seguridad y defensa están consolidadas a lo largo y ancho del continente, los soportes legales de estas aún se encuentran en construcción. Además, la gran mayoría de leyes o decretos sobre defensa nacional tiene más de diez años de antigüedad, lo cual indica un atraso o la poca importancia del tema.

En este mismo sentido, se identifica que la gran mayoría de las políticas analizadas corresponden a posiciones gubernamentales y no estatales. Esto disminuye la probabilidad de continuidad de las agendas de 
seguridad y defensa. Junto con los procesos socio-políticos que ha afrontado la región en los últimos veinte años, la falta de continuidad de las políticas hace prever que habrá una precariedad en las condiciones para aplicar los conceptos estratégicos del poder aéreo. Frente la homogénea situación de seguridad y defensa de la región, en la que las principales amenazas son el narcotráfico, el terrorismo, el crimen organizado y las condiciones socio-económicas de la población, las legislaciones no muestran la homogeneidad y multidimensionalidad necesarias.

Es poco el análisis de las condiciones en las que se encuentran las diferentes políticas públicas que hay en la región. Esto se debe principalmente a la ausencia de un direccionamiento estatal del planteamiento estratégico. En vez de esto, se encuentran las diferentes pretensiones de gobierno, lo que indica una fluctuación de los intereses y de las condiciones de aplicación de la política con cada cambio de gobierno.

Es necesario precisar que no se ha identificado con claridad o suficiencia el reconocimiento de la importancia de la superioridad aérea en el mundo, y de su conceptualización en el continente suramericano, por parte de los decisores políticos. Quienes formulan las políticas en el área del poder aéreo están en la necesidad de establecer como prioridad, eje o pilar estratégico, la superioridad aérea. Esta es una herramienta clave de disuasión y contención de las amenazas en los escenarios de conflictos armados inter e intra-estatales.

Sin embargo, los casos de Brasil, Chile, Venezuela y Colombia nos permiten inferir algunos criterios que definen al concepto de superioridad aérea. Así pues, la actuación de las mismas fuerzas militares, y en especial las fuerzas aéreas, de estos países se ha desarrollado sobre el supuesto de la importancia de conseguir la superioridad aérea.

Más allá de identificar que en ninguna de las políticas analizadas se hace referencia a la superioridad aérea, o que el desarrollo armamentístico en el escenario aeronáutico es evidente y que de allí se desprende la hipótesis de que sí hay una lucha por el objetivo de la superioridad aérea, se debe resaltar que existe la necesidad primaria de reformular muchas de las políticas públicas en Suramérica enfocadas en la seguridad y defensa. Es necesario alejar esas políticas de pretensiones gubernamentales y elevar la seguridad y la defensa a la categoría de interés estatal, con miras a la salvaguarda de los intereses nacionales. La prioridad debe ser realizar una construcción colectiva no solo de visiones, sino de las mismas políticas, de planteamientos estratégicos regionales, para fortalecer la lucha contra las amenazas que se deben afrontar.

Esto nos lleva a plantear la necesidad de consolidar escenarios de discusión regional para fomentar la cooperación en seguridad y defensa. Igualmente, debemos resaltar la necesidad de propender por la seguridad multidimensional ante las amenazas y para garantizar los intereses nacionales. En este aspecto, se destaca la política del Uruguay. Esta puede servir de inicio para la construcción de escenarios de seguridad multidimensional y ser un ejemplo de que una política pública puede atender a un interés colectivo regional si se proponen y apoyan los escenarios idóneos para que los interlocutores tengan la confianza necesaria y desarrollen coherentemente los procesos que garantizan la seguridad.

A pesar de las falencias en la consolidación de los criterios propios del poder aéreo, el avance y actualización constante de las políticas de seguridad en la región son el primer paso para lograr la consolidación de una política de defensa y seguridad regional. Dentro de esta política, la superioridad aérea debe ocupar un lugar importante con el fin de garantizar la paz y seguridad, no solo de cada uno de los países, sino de la región en su conjunto.

\section{Referencias bibliográficas}

Aguirre, L. M. (2006). Las relaciones entre América Latina y Estados Unidos: balances y perspectivas. En A. Borón y G. Lechini, Política y movimientos sociales en un mundo hegemónico (pp. 37-50). Consejo Latinoamericano de Ciencias Sociales.

Andrews, W. F. (1995). The Luftwaffe and the Battle for Air Superiority. Blueprint or Warning? Air Power Journal, 9(3), 
6-15. https://dialnet-unirioja-es.ezproxy.javeriana.edu. co/servlet/articulo?codigo $=6967091$

Asamblea Nacional de la República Bolivariana de Venezuela. (2002). Ley Orgánica de Seguridad de la Nación. Asamblea Nacional de la República Bolivariana de Venezuela.

Congreso de la Nación Paraguaya. (1998, 11 de abril). Ley 1337 de 1998. De defensa nacional y seguridad interna. https:// pdba.georgetown.edu/Security/citizensecurity/paraguay/ leyes/1.pdf

Corum, J. S. (2019). Los teóricos de la guerra aérea (1918-1939). Desperta Ferro: Contemporánea, 34, 62-65. https://dial net.unirioja.es/servlet/articulo?codigo $=6967091$

Facultad Latinoamericana de Ciencias Sociales. (2016, octubre). La riesgosa política del gobierno para las fuerzas armadas. http://flacso.org.ar/wp-content/uploads/2016/10/la_ riesgosa_politica_del_gobierno_para_las_ffaa.pdf

Fitzgerald, V. (2009). La distribución de ingresos y rentas en América Latina durante el siglo xx: un estudio inicial. Cuadernos Económicos Revista ICE, 78, 29-59. http://dx. doi.org/10.15446/cuad.econ.v36n72.65819

Giaccaglia, C. (2017). Las nuevas teorías del poder aéreo y su influencia en el diseño de la campaña: evolución de los conceptos de Boyd y Warden. http://www.cefadigital.edu.ar/ bitstream/1847939/1133/1/RESGA\%20241\%20GIACCA GLIA.pdf

Giudice, V. (2005). Teorías Geopolíticas. Gestión en el Tercer Milenio, Revista de Investigación de la Facultad de Ciencias Administrativas, UNMSM, 8(15), 19- 23. https://doi. org/10.15381/gtm.v8i15.9691

Gobierno de la República Federativa de Brasil. (2012). Libro Blanco de Defensa Nacional. Gobierno de la República Federativa de Brasil.

Gobierno de la República Oriental del Uruguay. (2014). Política de Defensa Nacional. Gobierno de la República Oriental del Uruguay.

Grupo Simbólico de Transporte Aéreo. (2018, 5 de marzo). Grupo Simbólico de Transporte Aéreo 346. http://www. grupo346.com.uy/boletin/comunes/Analisis_evolu cion_doctrina_operaciones_aereas.pdf

Hadmann, F. N. (2019). A influência dos Arquitetos do Poder Aéreo na Estruturação de Forças Aéreas. Revista Defesa e Segurança, 3, 29-48. http://revistaeletronica.fab.mil.br/ index.php/afa/article/download/99/108

Jordán Enamorado, J. (2016). El debate sobre la primacía del poder aéreo: un recorrido histórico. Bie3: Boletín IEEE, 4, 1039-1077. https://dialnet.unirioja.es/servlet/ articulo? codigo $=6029307$
Ministerio de Defensa de la República de Bolivia. (2004). Libro Blanco de Defensa de la República de Bolivia. Instituto Geográfico Militar.

Ministerio de Defensa del Perú. (2005). Libro Blanco de Defensa Nacional. Ministerio de Defensa del Perú.

Ministerio de Defensa Nacional. (2011). Política Integral de Seguridad y Defensa para la Prosperidad. Imprenta Nacional de Colombia.

Ministerio de Defensa Nacional de Argentina. (1998). Libro Blanco de la República Argentina. República Argentina.

Navarrete Anderson, F. G. (2019). El Control del Espacio Aéreo en el Perú Como Factor Contribuyente para la Defensa Nacional, año 2017 [Tesis de Maestría no publicada]. Centro de Altos Estudios Nacionales, Lima.

Ochoa, O. A. (2013). Plan Colombia: una lectura retrospectiva. Panorama, 7(12), 9-22. https://journal.poligran.edu.co/ index.php/panorama/article/view/366

Peixoto de Oliveira, R. (2014). (Re) Configuración o vieja configuración geopolítica hemisférica: La integración de la Región Andina en el comienzo del siglo xxı. Revista Andina de Estudios Políticos, 4(2), 1-17. https://ds pace.unila.edu.br/bitstream/handle/123456789/2593/ Peixoto\%20\%26\%20Centuri\%C3\%B3n.pdf?sequence= $1 \&$ isAllowed=y

Piglia, M. (2018). Poder aéreo y concurrencia regulada: las raíces de la política aérea argentina, 1935-1966. Revista de Gestión Pública, 7(1), 77-105. https://www.researchgate. net/publication/329399458_PODER_AEREO_Y_CONCU RRENCIA_REGULADA_LAS_RAICES_DE_LA_POLITICA_ AEREA_ARGENTINA_1935-1966

Pizarro, J. M. (2002). Poder Aéreo en América del Sur. Las Fuerzas Aéreas Latinoamericanas en el siglo 21. Tecnología Militar, 30-36. https://www.insumisos.com/lecturasin sumisas/Poder\%20Aereo\%20en\%20Am\%E9rica\%20 del\%20Sur.pdf

Presidente de la República del Perú. (2018, 21 de marzo). Decreto Supremo 012-2017-DE. http://busquedas.elperuano.pe/normaslegales/decreto-supremo-que-aprue ba-la-politica-de-seguridad-y-defen-decreto-supremon-012-2017-de-1600032-1/

Renouvin, P. (1990). La Primera Guerra Mundial. Oikos-tau.

República de Chile. (2010). Libro de la Defensa Nacional de Chile. Gobierno de Chile.

República del Ecuador. (2005). Libro Blanco del Ecuador. República del Ecuador.

Reyes Segovia, M. E. \& Urbina Rodríguez, H. A. (2014). La política exterior de la República Popular de China para la 
cuenca del Pacífico. Una nueva correlación de fuerzas para América Latina. Perspectivas para Centroamérica, periodo 2007-2012. Universidad De El Salvador. Facultad De Jurisprudencia Y Ciencias Sociales. Escuela De Relaciones Internacionales.

Saíz, M., \& Saíz, D. (2012). El inicio de la psicología aplicada a la aviación: desde el principio del siglo xx a la década de los años 30. Revista de Historia de la psicología, 33(4), 7-36. https://dialnet.unirioja.es/descarga/articu lo/5452522.pdf

Sanguinetti, P., \& Villar, L. (2012). Patrones de desarrollo en América Latina: ¿Convergencia o caída en la trampa del ingreso medio? Coyuntura Económica, 42(2), 155-188. https://scioteca.caf.com/handle/123456789/231

Serrano, J. M. (2017). La industria de guerra alemana como elemento de planificación estratégica durante la Segunda Guerra Mundial. Revista de Estudios en Seguridad Internacional, 3(2), 77-96. http://dx.doi.org/10.18847/1.6.5

Siminic Ossio, I. (2018). Fuerza Aérea de Chile, historia e Identidad Nacional. Revista Política y Estrategia, 115, 172182. https://dialnet.unirioja.es/servlet/articulo?codi go $=5182662$
Simonetta, J. C. (2016, 1 de septiembre). Argentina y la imposibilidad de defender su espacio aéreo. http://www. seguridadinternacional.es/?q=es/content/argenti na-y-la-imposibilidad-de-defender-su-espacio-a\%C3 \%A9reo

Sodupe, K. (1991). La teoría de la disuasión: un análisis de las debilidades del paradigma estatocéntrico. Afers Internacionals, 22, 53-79. https://core.ac.uk/download/ pdf/39007618.pdf

Tapia, L., \& Gavino, L. (2019). Visión geopolítica del Ecuador en el período 2006-2016: Su influencia en la Seguridad y Defensa [Tesis de Maestría no publicada] Instituto de Altos Estudios Nacionales, Quito.

Vitelli , M. (2015). La identidad estratégica de Brasil: política de defensa. Colombia Internacional, 85, 141-169. http://dx. doi.org/10.7440/colombiaint85.2015.05

Widnall, S. E. (1995). State of the Air Force. Airpower Journal, 9(1), 4-14.

Zuinaga, S. (2015). El enfoque de la geopolítica en el contexto de las relaciones internacionales en el nuevo milenio. Revista Venezolana de Análisis de Coyuntura, 21 (1), 11-32. https://www.redalyc.org/articulo.oa?id=36442240002 